\title{
Efetividade de uma intervenção fisioterapêutica cognitivo-motora em idosos institucionalizados com comprometimento cognitivo leve e demência leve
}

\author{
Effectiveness of a cognitive-motorphysiotherapeutical therapy \\ intervention in institutionalized older adults \\ with mild cognitive impairment and mild dementia
}

Alessandra Vieira Menezes ${ }^{1}$

Alessandra da Silva de Aguiar ${ }^{1}$

Elysama Fernandes Alves ${ }^{1}$

Layse Biz de Quadros ${ }^{1}$

Poliana Penasso Bezerra ${ }^{1}$

${ }^{1}$ Universidade Federal

de Santa Catarina. Rod.

Governador Jorge Lacerda

3201/Km 35.4, Jardim

das Avenidas. 88900-000

Araranguá SC Brasil.

alessandra.v.me@gmail.com
Abstract The aim of this study was to investigate the effectiveness of four months of cognitive-motor physiotherapy intervention, with a single weekly visit, on cognitive function, mobility and functional independence of institutionalized elderly individuals with mild cognitive impairment and mild dementia. In a controlled clinical trial, 15 subjects were allocated to experimental and control groups. Regarding the assessment of the subjects the following instruments were applied: Mini-Mental State Examination, Clinical Dementia Rating Scale, Verbal Fluency Test and Frontal Assessment Battery for analyzing cognitive functions; Berg Balance Scale and Timed Up and Go Test to verify mobility, and; Barthel Scale and Pfeffer Index for measurement of functional independence. The statistical procedures involved the application of Student's t-test with a 5\% significance threshold. With respect to the results, the experimental group performed better only in mobility-related tests at the end of the intervention $(p<0.05)$. The proposed intervention showed efficacy on mobility, but not on cognition and functional independence. The short period of time and low weekly basis may be related to the results obtained.

Key words Mild cognitive impairment, Long-stay institutions for the elderly, Exercise therapy
Resumo O objetivo desta pesquisa foi verificar a efetividade de quatro meses de intervenção fisioterapêutica cognitivo-motora, com frequência semanal única, na cognição, mobilidade e independência funcional de idosos com comprometimento cognitivo e demência leves residentes em instituição. Sob um ensaio clínico controlado, 15 sujeitos foram alocados entre grupo experimental (GE) e controle (GC). Para avaliação dos sujeitos foram aplicados os instrumentos: Mini-Exame do Estado Mental, Teste de Fluência Verbal e Bateria de Avaliação Frontal para análise das funções cognitivas; Escala de Equilíbrio Funcional de Berg $e$ Teste Timed Up and Go para verificação da mobilidade, e Escala de Barthel e Índice de Pfeffer para mensuração da independência funcional. $O$ procedimento estatístico envolveu a aplicação do teste t de Student, sob significância de 5\%. Quanto aos resultados, o GE apresentou melhor desempenho apenas nos testes relacionados à mobilidade ao final da intervenção $(p<0,05)$. A intervenção proposta apresentou eficácia sobre a mobilidade, mas não na cognição e independência funcional. O curto período de tempo e a baixa frequência semanal podem estar relacionados aos resultados obtidos.

Palavras-chave Comprometimento cognitivo leve, Instituição de longa permanência para idosos, Terapia por exercício 


\section{Introdução}

Com o envelhecimento da população ${ }^{1}$, as instituições de longa permanência (ILP) têm um papel crucial no suporte ao idoso. Apesar de, historicamente, as ILPs serem caracterizadas como locais onde vivem pessoas que exigem cuidados, idosos sadios têm decidido, mais frequentemente, viver nessas instituições por diferentes motivos (contato social mais intenso ou até mesmo aproveitamento dos serviços oferecidos na ILP, como alimentação adequada e balanceada, atividade física e recreativa e suporte médico especializado). As ILPs são geralmente divididas em áreas com base na dependência dos residentes em suas atividades diárias. Em geral, os residentes mais dependentes são aqueles que necessitam de mais gastos com seus cuidados ${ }^{2}$.

O declínio cognitivo é um dos principais motivos para institucionalização de idosos e, com frequência, contribui para o aumento da dependência na ILP. Uma vez detectado, o comprometimento cognitivo precisa de acompanhamento ${ }^{3}$. A maioria das demências não tem cura, porém há casos que são potencialmente reversíveis ${ }^{4}$. Os tipos mais comuns de demência são a doença de Alzheimer, demência vascular, demência frontotemporal e demência dos corpos de Lewy ${ }^{5,6}$. Outra condição clínica importante é o comprometimento cognitivo leve, definido como um estado intermediário entre o envelhecimento normal e a demência, cuja característica principal é o comprometimento da memória episódica, sem outros déficits cognitivos e funcionais, representando um risco aumentado de desenvolver demência de Alzheimer ${ }^{7}$. Apesar da concepção de que os indivíduos nos estágios iniciais de deficiência cognitiva não apresentem deficiência em atividades diárias, as evidências sugerem que o desempenho destas, quando complexas, com maior exigência cognitiva, podem ser afetadas ${ }^{8}$. A avaliação multidisciplinar e o planejamento das futuras ações de saúde são questões importantes, que contribuem para detecção precoce do comprometimento cognitivo em ILPs e intervenções interdisciplinares eficazes na referida população ${ }^{3}$.

Existem instrumentos simples que podem ser utilizados para rastreamento cognitivo de diferentes tipos de populações, incluindo aqueles que vivem em ILP. O Mini-Exame do Estado Mental $(\mathrm{MEEM})^{9}$ tem sido largamente estudado em populações diferentes, inclusive na brasileira. Seu desempenho está particularmente ligado à escolaridade, tendo pontos de corte bem estabelecidos para essa população ${ }^{10}$. No Brasil, as recomen- dações para o diagnóstico de demência foram elaboradas pelos membros do Departamento de Neurologia Cognitiva e do Envelhecimento da Academia Brasileira de Neurologia e baseiam-se nos avanços no conhecimento principalmente da avaliação neuropsicológica, de biomarcadores para demência e exames de imagem verificados nos últimos anos ${ }^{11}$.

Mesmo com os benefícios da prática da atividade física sobre as funções cognitivas e funcionais dos idosos já terem sido amplamente difundidos, a revisão sistemática elaborada por Crocker et al. ${ }^{12}$ demonstra que evidências na população idosa institucionalizada ainda são insuficientes. Além disso, os benefícios de intervenções com exercícios físicos em idosos com comprometimento cognitivo leve e estágio inicial de demência ainda são incertos ${ }^{13-15}$.

Sendo assim, o objetivo do presente estudo foi verificar a efetividade de uma intervenção fisioterapêutica cognitivo-motora na cognição, mobilidade e independência funcional de idosos com comprometimento cognitivo leve e demência leve, residentes em uma instituição de longa permanência.

\section{Metodologia}

\section{Delineamento}

Este estudo foi realizado após aprovação do Comitê de Ética em Pesquisa do Centro de Hematologia e Hemoterapia de Santa Catarina HEMOSC, estando de acordo com a resolução do Conselho Nacional de Saúde 466/12. Os idosos e responsáveis foram informados a respeito dos objetivos, riscos e procedimentos envolvidos na pesquisa, e aqueles que aceitaram participar assinaram o termo de consentimento livre e esclarecido. A pesquisa foi realizada no primeiro semestre de 2015.

Trata-se de uma pesquisa empírica quantitativa do tipo ensaio clínico com dois grupos independentes: grupo experimental (GE) e grupo controle (GC). Os participantes foram selecionados em uma ILP da cidade de Araranguá/SC, por meio da técnica de amostragem não probabilística intencional, onde todos os indivíduos aptos e que contemplaram os critérios delimitados foram inseridos.

A Figura 1 apresenta o fluxograma referente à seleção do público-alvo e ao processo de composição dos grupos. Os critérios de inclusão foram: sujeitos de ambos os sexos, com idade superior a 
60 anos, com comprometimento cognitivo leve ou demência leve, independência funcional para ortostatismo e deambulação, e sedentários. Avaliação cognitiva por meio do MEEM, com escores inferiores ao ponto de corte estipulado por Brucki et al. ${ }^{10}$, foi utilizada para caracterizar o declínio cognitivo. Os idosos incluídos apresentavam na Escala de Avaliação Clínica da Demência ( $C l i$ nical Dementia Rating - CDR $)^{16}$ classificação 0,5 ou 1,0, caracterizando o comprometimento cognitivo leve e a demência leve. Foram excluídos os participantes com distúrbios neurológicos e psiquiátricos, amaurose e cardiopatia grave.

Diante dos critérios estipulados, foram incluídos 15 indivíduos com idade média de 75,46 \pm 8,02 anos (intervalo de confiança: 71,02 a 79,91 anos). A alocação dos sujeitos se deu a partir do interesse inicial dos idosos em participar da pesquisa. Selecionados todos os interessados que contemplaram os critérios de participação, realizou-se o processo de alocação onde foi estabelecida uma divisão aleatória, mas respeitando a dimensão dos estratos. Diante disso, pós-divisão, o grupo experimental (GE) apresentou 7 sujeitos e o grupo controle (GC) 8.

\section{Procedimentos metodológicos}

Sobre os procedimentos metodológicos, os idosos foram divididos em grupo experimental, participantes do programa de exercícios multicomponentes com estimulação cognitivo-motora durante 4 meses e com frequência de uma

sessão semanal de uma hora de duração, e grupo controle, submetido à avaliação longitudinal de acompanhamento sem aplicação da referida intervenção. A terapia medicamentosa de todos os participantes foi registrada.

Sobre os exercícios, as sessões eram iniciadas com alongamentos seguido de exercícios de fortalecimento muscular. $\mathrm{Na}$ sequência, eram realizadas atividades que estimulassem de forma lúdica o desempenho funcional, objetivando a dissociação de cinturas, a coordenação motora, o equilíbrio, a propriocepção e a cognição, em especial a memória. Ao final, era realizado treino de marcha (Quadro 1). Os exercícios foram realizados em uma salão amplo, com boa iluminação, equipado com cadeiras e equipamento de som.

Em todas as sessões havia músicas variadas, com o objetivo de promover ativação sensorial auditiva. A pressão arterial e a frequência respiratória foram aferidas antes e ao término de cada sessão para evitar riscos associados à pesquisa. Esta intervenção fisioterapêutica baseia-se na proposta de Martins et al. ${ }^{17}$.

\section{Instrumentos de avaliação}

Quanto à avaliação dos sujeitos, foram aplicados os seguintes instrumentos: MEEM $^{9-11}$, Teste de Fluência Verbal Semântica ${ }^{18}$ e Bateria de Avaliação Frontal ${ }^{19}$ para análise das funções cognitivas; Escala de Equilíbrio Funcional de Berg $^{20}$ e Teste Timed Up and Go (TUG) ${ }^{21}$ para verificação da mobilidade, e, Escala de Barthel modificada ${ }^{22}$ e Índice de Pfeffer $^{23}$ para mensuração da independência funcional dos participantes.

Os instrumentos de análise cognitiva aplicados nesta pesquisa abordaram as diversas funções cognitivas: enquanto que o MEEM avalia orientação para tempo, local, atenção, cálculo e praxia visuo-construtiva, o Teste de Fluência Verbal abrange habilidades de memória semântica e linguagem e a Bateria de Avaliação Frontal exige ativação das funções executivas.

A mensuração da mobilidade dos participantes se deu por meio da Escala de Equilíbrio Funcional de Berg e do teste TUG. A Escala de Equilíbrio Funcional de Berg é uma escala composta por 14 itens envolvendo tarefas funcionais específicas em diferentes situações e bases de apoio, e que analisa o equilíbrio estático e dinâmico dos sujeitos. O TUG é um importante teste que mensura a mobilidade de idosos, no qual o sujeito levanta de uma cadeira, anda três metros e volta à mesma cadeira. Neste teste foi contabilizado o tempo gasto para realizar a atividade. 
Quadro 1. Sessão de uma hora de duração do programa de exercícios multicomponentes com estimulação cognitivo-motora.

\begin{tabular}{|l|l|}
\hline $\begin{array}{l}\text { Exercícios de alongamento } \\
\text { muscular }\end{array}$ & $\begin{array}{l}\text { Os exercícios de alongamento foram realizados de maneira suave e no limite } \\
\text { de cada idoso, estando este em pé. Os músculos envolvidos foram: músculos } \\
\text { da região cervical, trapézios, paravertebrais, peitorais, deltoides, bíceps, tríceps, } \\
\text { flexores e extensores do punho, quadríceps, isquiotibiais e tríceps sural. Cada } \\
\text { alongamento foi realizado durante 30 segundos. }\end{array}$ \\
\hline $\begin{array}{l}\text { Exercícios de fortalecimento } \\
\text { muscular }\end{array}$ & $\begin{array}{l}\text { Inicialmente o fortalecimento muscular foi realizado em cadeia cinética } \\
\text { aberta com auxílio de pesos com 0,5 a 1,0 Kg. Estando o idoso sentado: os } \\
\text { exercícios foram de flexão e extensão das articulações do ombro, cotovelo e } \\
\text { joelho. Estando o idoso em pé: os exercícios foram flexão de joelho, flexão de } \\
\text { quadril, extensão de quadril e elevação lateral do membro inferior. Em seguida } \\
\text { eram realizados, em cadeia cinética fechada, exercícios de flexão plantar e de } \\
\text { agachamento sem adição de carga. Realizado 2 séries de 10 repetições de cada } \\
\text { exercício. }\end{array}$ \\
\hline $\begin{array}{l}\text { Exercícios de atividades } \\
\text { funcionais }\end{array}$ & $\begin{array}{l}\text { Exercício de transferência de levantar-se e sentar-se sem a utilização das mãos; } \\
\text { exerćcio enfatizando a rotação de tronco realizado nas posições sentado e em } \\
\text { pé com o uso de bolas e bastões. Realizadas 2 séries de 10 repetições de cada } \\
\text { exercício. }\end{array}$ \\
\hline $\begin{array}{l}\text { Exercícios de treino de } \\
\text { marcha } \\
\text { motora com tarefa cognitiva } \\
\text { associada }\end{array}$ & $\begin{array}{l}\text { Exercícios de passar a bola em roda falando substantivos iniciados com } \\
\text { determinadas letras do alfabeto ou o nome da pessoa ao lado; caminhar } \\
\text { realizando tarefas associadas, como retirando objetos de uma sacola ou } \\
\text { cantando uma música; memorizar e imitar uma atividade doméstica ou animal } \\
\text { citada anteriormente pelo idoso posicionado ao lado e acrescentar mais uma. }\end{array}$ \\
\hline $\begin{array}{l}\text { Marcha sobre superfície instável (colchão); passadas laterais com deslocamento } \\
\text { em linha reta e em curvas, com e sem obstáculos, com distratores cognitivos } \\
\text { (contagens, memorizações, identificando dias da semana, mês e ano), em } \\
\text { circuitos cada vez mais complexos. }\end{array}$ \\
\hline
\end{tabular}

Por fim, para a mensuração das atividades funcionais utilizou-se um instrumento que verifica a rotina dos participantes nas atividades básicas da vida diária (Escala de Barthel modificada) e outro que analisa a independência dos sujeitos nas atividades instrumentais da vida diária (Índice de Pfeffer). Apesar da amostra ser constituída por idosos residentes em instituições, optou-se pelo Índice de Pfeffer, pois o mesmo analisa atividades complexas que, por mais que não sejam realizadas pelos participantes em seu dia-a-dia, lhe dão a opção de responder "não realiza mas conseguiria realizar", sem qualquer prejuízo de mensuração.

Em relação aos instrumentos aplicados, todos se encontram validados à língua portuguesa e são aplicáveis à referida população.

\section{Análise estatística}

Os dados foram analisados pelo programa estatístico SPSS versão 21. Os dados descritivos foram detalhados por meio de média, desvio-padrão e intervalo de confiança, (estipulado em 95\%). Os critérios paramétricos de normalidade e homogeneidade de variâncias foram analisados pelo teste de normalidade de Shapiro-Wilk.

A estatística inferencial foi realizada por meio de análises transversais e longitudinais. As variáveis apresentaram distribuição normal, então, a comparação intergrupo inicial e final deu-se pelo teste $t$ de Student para amostras independentes, o teste de qui-quadrado utilizado para comparar a proporção de participantes e a comparação intra-grupo inicial e final deu-se pelo teste $t$ de Student para amostras emparelhadas. Para todas 
as análises, adotou-se uma significância de 5\% (p $<0,05)$.

\section{Resultados}

Esta pesquisa abordou idosos com comprometimento cognitivo e demência leves residentes em ILP divididos entre os grupos GE e GC. Por meio do teste $t$ Student para amostras independentes verificou-se que os indivíduos não apresentavam diferenças significativas em relação à idade ( $\mathrm{p}=$ 0,77 ), sendo que os idosos do GE apresentavam média de idade de 76,14 \pm 9,17 anos (intervalo de confiança: 67,65 a 84,62 anos) e os idosos do grupo controle 74,00 \pm 7,61 anos (intervalo de confiança: 66,95 a 81,04 anos). Também não foi verificado diferença significativa em relação ao tempo de institucionalização $(p=0,77)$, sendo que os idosos do GE apresentavam média de tempo de 79,42 \pm 89,33 meses (intervalo de confiança: $-3,19$ a 162,04 meses) e os do grupo controle 41,57 \pm 45,69 meses (intervalo de confiança: $-0,68$ a 83,82 meses). O total de sujeitos por grupo $(\mathrm{p}=0,51)$ e a proporção de homens e mulheres nestes $(\mathrm{p}=0,38)$ também apresentaram similaridades entre GE e GC.
A perda amostral desta pesquisa foi da ordem de $13,33 \%$ no GE (2 idosos), sendo que um deixou de residir na instituição e outro apresentou intercorrência médica necessitando de internação hospitalar, sendo excluídos na análise dos dados.

A Tabela 1 detalha o comportamento dos grupos (média, desvio-padrão e intervalo de confiança de $95 \%$ para média) ao longo do período de acompanhamento (momento inicial e final). A análise transversal referente aos valores iniciais comprovou similaridade entre os grupos no momento inicial em relação ao MEEM ( $\mathrm{p}=$ $0,15)$, Teste de Fluência Verbal $(p=0,32)$, Bateria de Avaliação Frontal ( $p=0,86)$, Escala de Equilíbrio Funcional de Berg $(\mathrm{p}=0,64)$, TUG ( $\mathrm{p}=$ $0,66)$, Escala de Barthel modificado $(\mathrm{p}=0,38)$ e Índice de Pfeffer $(\mathrm{p}=0,16)$.

$\mathrm{Na}$ análise do efeito da intervenção sobre as variáveis cognitivas, evidenciou-se que quatro meses de assistência terapêutica não proveram melhora significativa do GE nos instrumentos $\operatorname{MEEM}(\mathrm{p}=0,15)$, Teste de Fluência Verbal $(\mathrm{p}=$ $0,14)$ e Bateria de Avaliação Frontal ( $\mathrm{p}=0,63)$.

Para verificação dos efeitos da intervenção sobre a mobilidade dos sujeitos, a análise demonstra que quatro meses de assistência tera-

Tabela 1. Comportamento dos grupos referente às variáveis cognição, mobilidade e funcionalidade nos momentos inicial e final.

\begin{tabular}{lrrr}
\hline & & Grupo controle & \\
\cline { 2 - 4 } & Momento inicial & Momento final & p \\
\hline MEEM & $18,28 \pm 2,75(15,74$ a 20,83$)$ & $15,42 \pm 4,31(11,43$ a 19,41$)$ & 0,11 \\
TFV & $5,02 \pm 2,25(3,43$ a 9,82$)$ & $5,77 \pm 2,62(4,65$ a 8,58$)$ & 0,42 \\
BAF & $5,00 \pm 3,65(1,62$ a 8,37$)$ & $5,88 \pm 3,94(1,12$ a 8,86$)$ & 0,85 \\
EEFB & $48,25 \pm 3,77(45,09$ a 51,40$)$ & $49,98 \pm 3,02(44,88$ a 50,63$)$ & 0,22 \\
TUG & $22,5 \pm 12,07(18,66$ a 30,55$)$ & $23,5 \pm 11,45(17,26$ a 28,15$)$ & 0,19 \\
EBM & $82,14 \pm 20,68(63,01$ a 101,27$)$ & $77,85 \pm 21,76(57,72$ a 97,98$)$ & 0,20 \\
IP & $11,00 \pm 5,53(5,87$ a 16,12$)$ & $11,33 \pm 5,02(5,44$ a 15,10$)$ & 0,32 \\
\hline & & Grupo experimental & \\
\hline MEEM & $14,42 \pm 3,90(10,81$ a 18,04$)$ & $16,85 \pm 5,39(12,86$ a 20,84$)$ & 0,15 \\
TFV & $4,89 \pm 2,25(2,04$ a 7,77$)$ & $6,42 \pm 1,10(4,18$ a 8,20$)$ & 0,14 \\
BAF & $6,00 \pm 2,64(3,55$ a 8,44$)$ & $7,22 \pm 3,45(5,02$ a 9,88$)$ & 0,63 \\
EEFB & $47,85 \pm 3,89(44,25$ a 51,25$)$ & $49,87 \pm 3,56(46,89$ a 52,85$)$ & $0,001^{*}$ \\
TUG & $22,06 \pm 8,89(11,43$ a 33,76$)$ & $19,87 \pm 6,61(11,38$ a 27,81$)$ & $0,04^{*}$ \\
EBM & $71,71 \pm 12,82(59,84$ a 83,57$)$ & $75,28 \pm 14,98(61,42$ a 89,14$)$ & 0,15 \\
IP & $12,71 \pm 12,00(9,95$ a 15,47$)$ & $11,77 \pm 5,57(5,98$ a 16,02$)$ & 0,18 \\
\hline
\end{tabular}

MEEM: Mini-Exame do Estado Mental; TFV: Teste de Fluência Verbal; BAF: Bateria de Avaliação Frontal; EEFB: Escala de Equilíbrio Funcional de Berg; TUG: Timed Up and Go; EBM: Escala de Barthel modificada; IP: Índice de Pfeffer. *Análise intragrupo nos momentos inicial e final por meio do teste $t$ de Student para amostras emparelhadas evidenciando diferença estatística significativa. Observação: Os dados estão dispostos em: média \pm desvio padrão (intervalo de confiança de 95\% para média). 
pêutica proveram melhora significativa no GE nos instrumentos Escala de Equilíbrio Funcional de Berg ( $\mathrm{p}=0,001$; intervalo de confiança de $95 \%$ para a diferença $=-3,46 \mathrm{a}-1,28)$ e TUG $(\mathrm{p}=$ 0,04 ; intervalo de confiança de $95 \%$ para a diferença $=0,34$ a 6,04).

Em relação aos efeitos da intervenção sobre a funcionalidade, as análises demonstraram que quatro meses de assistência não proveram melhora significativa no GE na Escala de Barthel Modificada $(\mathrm{p}=0,15)$ e no índice de Pfeffer ( $\mathrm{p}$ $=0,18$ ).

No GC, as análises demonstraram que após quatro meses de acompanhamento não houve modificação nos resultados das avaliações obtidas por meio dos instrumentos MEEM ( $\mathrm{p}=$ $0,11)$, Teste de Fluência Verbal $(p=0,42)$, Bateria de Avaliação Frontal $(p=0,85)$, Escala de Equilíbrio Funcional de Berg $(\mathrm{p}=0,22)$, TUG $(\mathrm{p}=$ $0,19)$, Escala de Barthel modificado $(\mathrm{p}=0,20)$ e Índice de Pfeffer $(\mathrm{p}=0,32)$.

\section{Discussão}

Idosos residentes em instituição com comprometimento cognitivo e demência leves submetidos à intervenção fisioterapêutica cognitivo-motora, aplicado com frequência de uma sessão semanal e desenvolvido durante quatro meses, apresentaram melhora significativa na mobilidade ao final da intervenção. O protocolo não mostrou eficácia sobre as funções cognitivas e a funcionalidade dos sujeitos, tendo obtido respostas similares nos grupos experimental e controle. Entender o comportamento de tais variáveis é fundamental para que os profissionais consigam realizar uma análise crítico-científica da situação do paciente e tomar a melhor decisão quanto à assistência a ser aplicada.

Em algumas instituições observa-se a recusa dos idosos em participar de programas de treinamento de força, equilíbrio e desempenho funcional, limitando a eficácia do programa ${ }^{24}$. O sedentarismo leva a uma reação cíclica, ocasionando maior morbidades e aderência cada vez menor dos idosos aos programas de intervenção com exercícios físicos ${ }^{25}$. Embora estudos usualmente reportem evidências que programas de reabilitação com sessões de 60 minutos, com frequências semanais de 2-3 vezes, estão relacionadas a melhores resultados em idosos com comprometimento cognitivo leve e demência leve ${ }^{13,26,27}$, parâmetros específicos para prescrição adequada ainda não são estabelecidos, especialmente em relação à duração, intensidade e tipo de atividade. Propomos um programa de frequência semanal única durante quatro meses que pode ser utilizado durante a implantação de programas fisioterapêuticos de exercícios em instituições onde residem idosos sedentários, que não têm o hábito de exercitar-se regularmente, favorecendo a aderência deles ao programa. Acredita-se que os benefícios observados em programas de reabilitação, mesmo aqueles com frequência semanal única, como o proposto no presente estudo, provocam interrupção do sedentarismo, revelandose um fator importante para descontinuar essa reação cíclica, promover uma adaptação à prática regular do exercício físico e melhorar a qualidade de vida de idosos institucionalizados ${ }^{25,28}$.

Em nosso estudo, a análise dos resultados obtidos com os instrumentos de avaliação cognitiva evidencia que a terapêutica aplicada no tempo de quatro meses de intervenção não foi efetiva. Não há consenso na literatura sobre os efeitos de intervenção cognitivo-motora nas funções cognitivas de idosos com e sem demência. Estudo de Martins et al. ${ }^{17}$ não evidenciou benefícios de uma intervenção fisioterapêutica cognitivo-motora, na qual se baseia a intervenção proposta neste estudo, em idosos institucionalizados sem declínio cognitivo, justificando os resultados pela falta de sensibilidade dos instrumentos de avaliação para constatar tênues diferenças promovidas pela intervenção na amostra sem deficiência cognitiva no tempo de intervenção proposto.

Enquanto alguns autores admitem que intervenção com exercícios físicos não influenciam as funções cognitivas, outros alegam que a grande dificuldade de provar os benefícios está relacionada ao pequeno número de estudos sobre o tema, dificuldade em controlar vieses metodológicos e curtos períodos de seguimento ${ }^{13,29,30}$. Revisão sistemática ${ }^{30}$ aponta que o exercício físico tem sido considerado uma estratégia de intervenção não farmacológica útil, capaz de melhorar a memória e funções executivas em idosos com declínio cognitivo, partindo da premissa dos efeitos do exercício sobre neurotransmissores e fator neurotrófico derivado do encéfalo ${ }^{31}$. Isto apoia ainda mais descobertas anteriores de que o treinamento pode induzir plasticidade cerebral, mesmo em adultos mais velhos com risco para desenvolver demência de Alzheimer ${ }^{32}$.

Então, levando-se em consideração estas pesquisas prévias que comprovam o benefício da intervenção cognitivo-motora em idosos com comprometimento da cognição, é possível argumentarmos que o tempo de intervenção 
associado a baixa frequência semanal (uma vez por semana) não foi suficiente para promover benefícios cognitivos na amostra. Ressaltamos que observando a média geral dos grupos nos momentos inicial e final, verifica-se um melhor desempenho no MEEM no grupo experimental após a intervenção e declínio no desempenho do grupo controle. Sendo assim, é possível que os instrumentos de avaliação utilizados não tenham sido sensíveis para constatar pequenas diferenças promovidas pela intervenção.

A mobilidade representa um dos fatores atrelados à inserção do idoso em ILP, pois, conforme demonstrado por Rosso et al..$^{33}$, as disfunções físicas tendem a diminuir o engajamento social e as redes de contatos das pessoas, dificultando o cuidado do familiar cuidador. Nesta pesquisa, os grupos experimental e controle apresentaram semelhança estatística para os valores iniciais de mobilidade. Em nosso estudo, a análise dos resultados obtidos com os instrumentos de avaliação de equilíbrio e mobilidade funcional evidencia que a terapêutica aplicada no tempo de quatro meses de intervenção foi efetiva, corroborando com a revisão sistemática de Gordon et al. ${ }^{34}$ e outros estudos em idosos com declínio $\operatorname{cognitivo~}^{27-29}$. Essas melhoras observadas podem estar relacionadas à otimização da marcha nos idosos, visto que programas de exercícios físicos multicomponentes podem ocasionar melhora na velocidade, comprimento da passada e ritmo dos movimentos do tronco nos com comprometimento cognitivo leve $\mathrm{e}^{35}$.

Em nosso estudo, não evidenciamos melhora significativa na independência funcional em atividades básicas do dia-a-dia, como o ato de vestirse, banhar-se, transferir-se, preservar continência e alimentar-se. Embora os resultados não tenham sido significativos, a observação das médias dos grupos nos momentos inicial e final indicam que o grupo experimental melhorou sua funcionalidade, enquanto que os idosos controles apresentaram redução da funcionalidade, aumentando a dependência nas atividades básicas de vida diária. As funções cognitivas representam um conjunto de funções corticais superiores envolvidas no planejamento, processamento e execução de ações vitais do dia-a-dia das pessoas. Estudos evidenciam que as melhoras obtidas na cognição podem estar relacionadas a outras na capacidade para realizar atividades básicas do dia-a-dia ${ }^{17}$. De acordo com Lawl et al. ${ }^{36}$, a intervenção cognitivo-motora pode influenciar diferentes domínios cognitivos e essa função é essencial para o desempenho adequado de tarefas funcionais. Por meio da inter- venção cognitivo-motora proposta foi fornecido aos idosos estímulos cognitivos, influenciando as funções cognitivas, e estímulos motores, influenciando a flexibilidade, a força muscular, a resistência, o equilíbrio, a propriocepção e a coordenação motora nas sessões de intervenção. Estudos evidenciam resultados positivos da intervenção cognitivo-motora, por meio de exercícios físicos multicomponentes, em pessoas com comprometimento cognitivo e demência leves ${ }^{27,28,36,37}$.

Não houve melhora observada nas atividades instrumentais de vida diária. Uma hipótese para a não observação de melhoras advindas da intervenção proposta seria o fato de muitos idosos não apresentarem o hábito de realizar as atividades instrumentais devido à rotina imposta pela própria instituição, podendo ser um fator de não percepção de modificação de tal desempenho. Além disso, as atividades instrumentais da vida diária são consideradas mais complexas que as básicas, por exigirem ativação cognitiva constante - em especial da área pré-frontal ${ }^{38}$.

A restrição no tamanho amostral foi influenciada diretamente pelos critérios de seleção dos sujeitos. Embora estudos comprovem um aumento no percentual de idosos residentes em instituições, estes usualmente caracterizam-se por necessitar de reabilitação intensiva, podendo estar em estágios terminais de doenças, muitos em condições crônico-neurodegenerativas, como com quadros de demência avançada, e com níveis elevados de dependência ${ }^{2}$. Assim, ao limitar o estudo a idosos com declínio cognitivo leve deambulantes e sedentários, restringiu-se esta pesquisa a uma pequena amostra de sujeitos, não sendo possível analisar separadamente aqueles com comprometimento cognitivo e demência leves. Além disso, é possível que tenha havido alguma interação medicamentosa não controlada nesta pesquisa. $\mathrm{O}$ fato da medicação dos idosos ter permanecido inalterada durante o período de intervenção pode ter atenuado essa interferência nos resultados.

Em suma, este estudo demonstrou benefícios de um programa de intervenção cognitivo-motor sobre a mobilidade de idosos com comprometimento cognitivo e demência leves, residentes em instituição, durante quatro meses de intervenção. O protocolo assistencial não foi eficaz sobre a função cognitiva e a funcionalidade dos participantes, refletindo a necessidade de novos estudos que analisem outras formas de estimulação cognitivo-motoras, com maior frequência semanal, aplicadas em tempo igual ou superior a quatro meses. 


\section{Colaboradores}

AV Menezes, AS Aguiar, EF Alves e LB Quadros trabalharam na coleta de dados, análise dos resultados e redação do artigo científico. PP Bezerra trabalhou na concepção, coleta de dados, análise dos resultados e redação do artigo científico.

\section{Referências}

1. Instituto Brasileiro de Geografia e Estatística (IBGE). Censo demográfico. IBGE: Rio de Janeiro; 2010.

2. Camarano AA, Kanso S. As instituições de longa permanência para idosos no Brasil. Rev Bras Estud Popul 2010; 27(1):232-235.

3. Jacinto AF, Brucki S, Porto CS, Martins MA, Nitrini R. Detection of cognitive impairment in the elderly by general internists in Brazil. Clinics 2011; 66(8):13791384.

4. Takada LT, Nitrini R, Caramelli P, Radanovic M, Anghinah R, Hartmann APBJ, Guariglia CC, Bahia VS, Nitrini R. Prevalence of potentially reversible dementias in a dementia outpatient clinic of tertiary university-affiliated hospital in Brazil. Arq Neuropsiquiatr 2003; 61(4):925-929.

5. Herrera E Jr, Caramelli P, Silveira AS, Nitrini R. Epidemiologic survey of dementia in a community-dwelling Brazilian population. Alzheimer Dis Assoc Disord 2002; 16(2):103-108.

6. Bottino CM, Azevedo Júnior D, Tatsch M, Hototian SR, Moscoso MA, Folquitto J, Scalco AZ, Bazzarella MC, Lopes MA, Litvoc J. Estimate of dementia prevalence in a community sample from São Paulo, Brazil. Dement Geriatr Cogn Disord 2008; 26(4):291-299.

7. Chaves ML, Camozzato AL, Godinho C, Piazenski I, Kaye J. Incidence of mild cognitive impairment and Alzheimer disease in Southern Brazil. J Geriatr Psychiatry Neurol 2009; 22(3):181-187.

8. Rodakowski J, Skidmore ER, Reynolds CF, Dew MA, Butters MA, Holm MB. Can performance on daily activities discriminate between older adults with normal cognitive function and those with mild cognitive impairment? J Am Geriatr Soc 2014; 62(7):1347-1352.

9. Folstein MF, Folstein SE, McHugh PR. "Mini-mental state". A practical method for grading the cognitive state of patients for the clinician. J Psychiatr Res 1975; 12(3):189-198

10. Brucki SMD, Nitrini R, Caramelli P, Bertolucci PH, Okamoto IH. Suggestions for utilization of the mini-mental state examination in Brazil. Arq Neuropsiquiatr 2003; 61(3B):777-781.

11. Frota NAF, Nitrini R, Damasceno BP, Forlenza O, Tosta ED, Silva AB, Junior EH, Magaldi RM. Critérios para o diagnóstico de doença de Alzheimer. Dement Neuropsychol 2011; 5(1):5-10.

12. Crocker T, Forter A, Young J, Brown L, Ozer S, Smith J, Green J, Hardy J, Burns E, Glidewell E, Greenwood DC. Physical rehabilitation for older people in long-term care. Cochrane Dababase Syst Rev 2013; 2:CD004294.

13. Rodakowski J, Saghafi E, Butters MA, Skidmore ER. Non-pharmacological interventions for adults with mild cognitive impairment and early stage dementia: An updated scoping review. Mol Aspects Med 2015; $43-$ 44:38-53.

14. Cooper C, Li R, Lyketsos C, Livingston G. Treatment for mild cognitive impairment: systematic review. $\mathrm{Br}$. J. Psychiatry 2013; 203(3):255-264. 
15. Huckans M, Hutson L, Twamley E, Jak A, Kaye J, Storzbach D. Efficacy of cognitive rehabilitation therapies for mild cognitive impairment (MCI) in older adults: working toward a theoretical model and evidence-based interventions. Neuropsychol Rev 2013; 23(1):63-80.

16. Morris JC. The Clinical Dementia Rating (CDR): Current version and scoring rules. Neurology 1993; 43(11):2412-2414

17. Martins AS, Freire RC, Martins LH, Berton B, Júnior SAO, Christofoletti G. Efeitos de uma terapia cognitivo-motora em idosos institucionalizados. Rev Bras Ativ Fis e Saúde 2014; 19(5):608-610.

18. Lezak MD. Neuropsychological assessment. $3^{\text {th }}$ ed. Oxford: Oxford University Press; 1995.

19. Dubois B, Slachevsky A, Litvan I, Pillon B. The FAB: A frontal assessment battery at bedside. Neurology 2000; 55(11):1621-1626.

20. Berg KO, Wood-Dauphine'e SL, Williams JI, Maki B. Measuring balance in theelderly: validation of an instrument. Can J Public Health 1992; 83(Supl. 2):S7-11.

21. Podsiadlo D, Richardson S. The 'Timed Up and Go': A test of basic functional mobility for frail elderly persons. J Am Geriatr Soc 1991; 39(2):142-148.

22. Mahoney FI, Barthel DW. Functional evaluation: the Bathel Index. Md. State Med. J 1965; 14:56-61.

23. Pfeffer RI, Kurosaki TT, Harrh CH, Chance JM, Filos S. Measurement of functional activities in older adults in the community. J Gerontol 1982; 37:323-329.

24. Benjamin K, Edwards N, Ploeg J, Legault F. Barriers to physical activity and restorative care for residents in long-term care: a review of the literature. J Aging Phys Act 2014; 22(1):154-165.

25. Gobbi LT, Oliveira-Ferreira MD, Caetano MJ, LiraniSilva E, Barbieri FA, Stella F, Gobbi S. Exercise programs improve mobility and balance in people with Parkinson's disease. Parkinsonism Relat Disord 2009; 15(Supl. 3):S49-52.

26. Cartucho MPM. Efeito de um programa de exercício físico multicomponente sobre a condição física e mental de idosos institucionalizados com demência leve [dissertação]. Porto: Universidade do Porto; 2013.

27. Kovács É, Sztruhár JCK, Karóczi Á, Korpos T, Gondos T. Effects of a multimodal exercise program on balance, functional mobility and fall risk in older adults with cognitive impairment: a randomized controlled single-blind study. Eur J Phys Rehabil Med 2013; 49(5): 639-648.

28. Quehenberger V, Cichocki M, Krajic K. Sustainable effects of a low-threshold physical activity intervention on health-related quality of life in residential aged care. Clin Interv Aging 2014; 9:1853-1864.

29. Andrade LP, Gobbi LT, Coelho FG, Christofoletti G, Riani Costa JL, Stella F. Benefits of multimodal exercise intervention for postural control and frontal cognitive functions in individuals with Alzheimer's disease: a controlled trial. J Am Geriatr Soc 2013; 61(11):1919-1926.
30. van Uffelen JG, Chin A, Paw MJ, Hopman-Rock M, van Mechelen W. The effects of exercise on cognition in older adults with and without cognitive decline: a systematic review. Clin J Sport Med 2008; 18(6):486-500.

31. Noble EE, Mavanji V, Little MR, Billington CJ, Kotz $\mathrm{CM}$, Wang C. Exercise reduces diet-induced cognitive decline and increases hippocampal brain-derived neurotrophic factor in CA3 neurons. Neurobiol Learn Mem 2014; 114:40-50.

32. Belleville S, Clément F, Mellah S, Gilbert B, Fontaine F, Gauthier S. Training-related brain plasticity in subjects at risk of developing Alzheimer's disease. Brain 2011; 134(Pt 6):1623-1634.

33. Rosso AL, Taylor JA, Tabb LP, Michael YL. Mobility, disability, and social engagement in older adults. J Aging Health 2013; 25(4):617-637.

34. Gordon AL, Phillipa AL, Jones RG, Forrester-Paton C, Mamo JP, Gladman JRF. A systematic mapping review of Randomized Controllet Trials (RCTs) in care homes. BMC Geriatr 2012; 12:31.

35. Doi T, Makizako H, Shimada H, Yoshida D, Tsutsumimoto K, Sawa R, Misu S, Suzuki T. Effects of multicomponent exercise on spatial-temporal gait parameters among the elderly with amnestic mild cognitive impairment (aMCI):preliminar results from a randomized controlled trial (RCT). Arch Gerontol Geriatr 2013;56(1):104-108.

36. Lawl LLF, Barnett F, Yaul MK, Gray MA. Effects of functional tasks exercise on older adults with cognitive impairment at risk of Alzheimer's disease: a randomised controlled trial. Age Ageing 2014; 43(6):813-820.

37. Fiatarone Singh MA, Gates N, Saigal N, Wilson GC, Meiklejohn J, Brodaty H, Wen W, Singh N, Baune BT, Suo C, Baker MK, Foroughi N, Wang Y, Sachdev PS, Valenzuela M. The Study of Mental and Resistance Training (SMART) study-resistance training and/or cognitive training in mild cognitive impairment: a randomized, double-blind, double-sham controlled trial. $J$ Am Med Dir Assoc 2014; 15(12):873-880.

38. Nikolova R, Demers L, Béland F. Trajectories of cognitive decline and functional status in the frail older adults. Arch Gerontol Geriatr 2009; 48(1):28-34.

Artigo apresentado em 18/09/2015

Aprovado em 15/12/2015

Versão final apresentada em 17/12/2015 
